Check for updates

Cite this: RSC Adv., 2019, 9, 6636

Received 5th February 2019

Accepted 12th February 2019

DOI: $10.1039 / \mathrm{c} 9 \mathrm{ra00976k}$

rsc.li/rsc-advances

\section{Electrophilic activation of nitroalkanes in efficient synthesis of 1,3,4-oxadiazoles $\uparrow$}

\author{
Alexander V. Aksenov, (D)*a Vladislav Khamraev, (D) a Nicolai A. Aksenov, (D) a \\ Nikita K. Kirilov, ${ }^{a}$ Dmitriy A. Domenyuk, ${ }^{b}$ Vladimir A. Zelensky ${ }^{\mathrm{b}}$ \\ and Michael Rubin (D)*ac
}

A novel methodology for general and chemoselective preparation of non-symmetric 1,3,4-oxadiazoles is developed. This unusual reaction proceeds via polyphosphoric acid-assisted activation of nitroalkanes towards nucleophilic attack with acylhydrazides.

\section{Introduction}

Bioisosteric to carboxylate and carboxamide functionalities, 1,3,4-oxadiazoles are often considered among other privileged heterocyclic scaffolds for drug discovery. ${ }^{1}$ Molecules possessing these key structural fragments have demonstrated a wide variety of important biological properties, including antibacterial, antimycobacterial, antifungal, insecticidal, herbicidal, anti-inflammatory, analgesic, anticonvulsant and anticancer activities. ${ }^{2}$ Several medicinal agents featuring this heterocyclic ring have been marketed, including anti-HIV drug Raltegravir, antihypertensive Nesapidil, and anti-cancer agent Zibotentan (Fig. 1). This heterocyclic building block was also used for the construction of chiral catalysts ${ }^{3}$ and metal-selective chemosensors. ${ }^{4}$ A symmetrically substituted version of this system (5) can be easily obtained by cyclo-condensation of hydrazine hydrate with a variety of carbonyl compounds. ${ }^{5}$ Assembly of 1,3,4-oxazoles 4 with two different substituents is much more challenging, as it involves the selective reaction of hydrazine with two different carbonyl compounds, or their synthetic equivalents, to obtain non-symmetric $N, N^{\prime}$-diacylhydrazide precursor 2 (Scheme 1). Such processes, especially when carried out in one-pot fashion, can be significantly complicated by side reactions involving trans-acylation of $N, N^{\prime}$-diacylhydrazide intermediates with excess of the second carbonyl compound and leading to the formation of a mixture of nonsymmetric (4) and symmetric (5) products. A variety of methods were suggested in an attempt to address this issue,

${ }^{a}$ Department of Chemistry, North Caucasus Federal University, 1a Pushkin St., Stavropol 355009, Russian Federation. E-mail: alexaks05@rambler.ru

${ }^{b}$ Department of General Practice Dentistry and Child Dentistry, Stavropol State Medical University, 310 Mira Street, Stavropol 355017, Russian Federation

'Department of Chemistry, University of Kansas, 1567 Irving Hill Road, Lawrence, KS 66045-7582, USA. E-mail: mrubin@ku.edu; Tel: +1-785-864-5071

$\dagger$ Electronic supplementary information (ESI) available. CCDC 1875720, 1875721 and 1875723. For ESI and crystallographic data in CIF or other electronic format see DOI: $10.1039 / \mathrm{c} 9 \mathrm{ra00976k}$ most of which are dealing with the moderation of the reactivity of the acylating reagent. ${ }^{6}$ In addition, different schemes involving cyclo-condensations of $N^{\prime}$-alkylidene acylhydrazides, ${ }^{7}$ or re-cyclizations of tetrazoles,${ }^{8}$ were also suggested. Herein we wish to report a new preparative method for selective assembly of non-symmetrically substituted 1,3,4-oxadiazoles employing nitroalkanes electrophilicity activated in the presence of polyphosphoric acid that serves as an acylating agent equivalent.

\section{Results and discussion}

For several years our group had a great interest in the development of novel acid-mediated cascade transformations of nitroalkenes and nitroalkanes targeting material science and medicinal chemistry applications. It was demonstrated that<smiles>COc1ccccc1N1CCN(CC(O)COc2cccc(-c3nnc(C)o3)c2)CC1</smiles>

Fig. 1 1,3,4-Oxazoles in drug discovery and medicinal chemistry. 


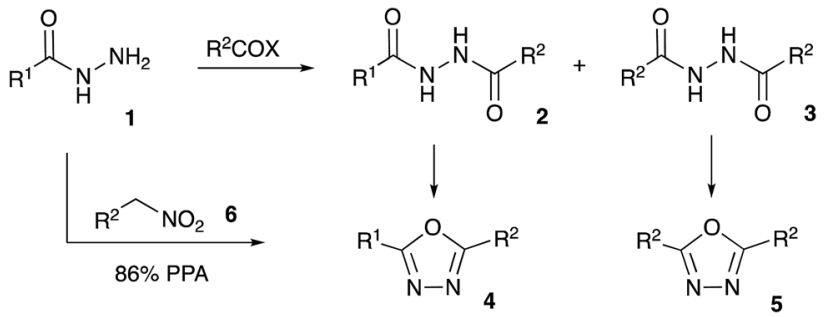

Scheme 1

nitroalkanes 6 dissolved in polyphosphoric acid (PPA) transform into phosphorylated nitronate 7 showing strong electrophilic properties. This unusual species can be used to design one-pot multi-step transformations involving various carbon-based nucleophiles. ${ }^{9}$ The utilization of nucleophilic amines was also demonstrated. ${ }^{10}$ Mechanistically, the latter process is related to the classical Nef reaction, ${ }^{11}$ employing aniline species 8 instead of water. This reaction provides imidinium ion $\mathbf{9}$, that can be further employed as a convenient building block for the synthesis of heterocyclic compounds: oxazoles 10, imidazoles 11, and diazines 12-13 (Scheme 2). ${ }^{10}$ We wondered about the possibility of employing acylhydrazides 1 as nucleophilic components en route to the 1,3,4oxazoles scaffolds. Indeed, such a nucleophilic attack to nitronate species 7 would afford (2-acylhydrazineyl)alkaniminium species $\mathbf{1 4},{ }^{12}$ that should be well suited for an

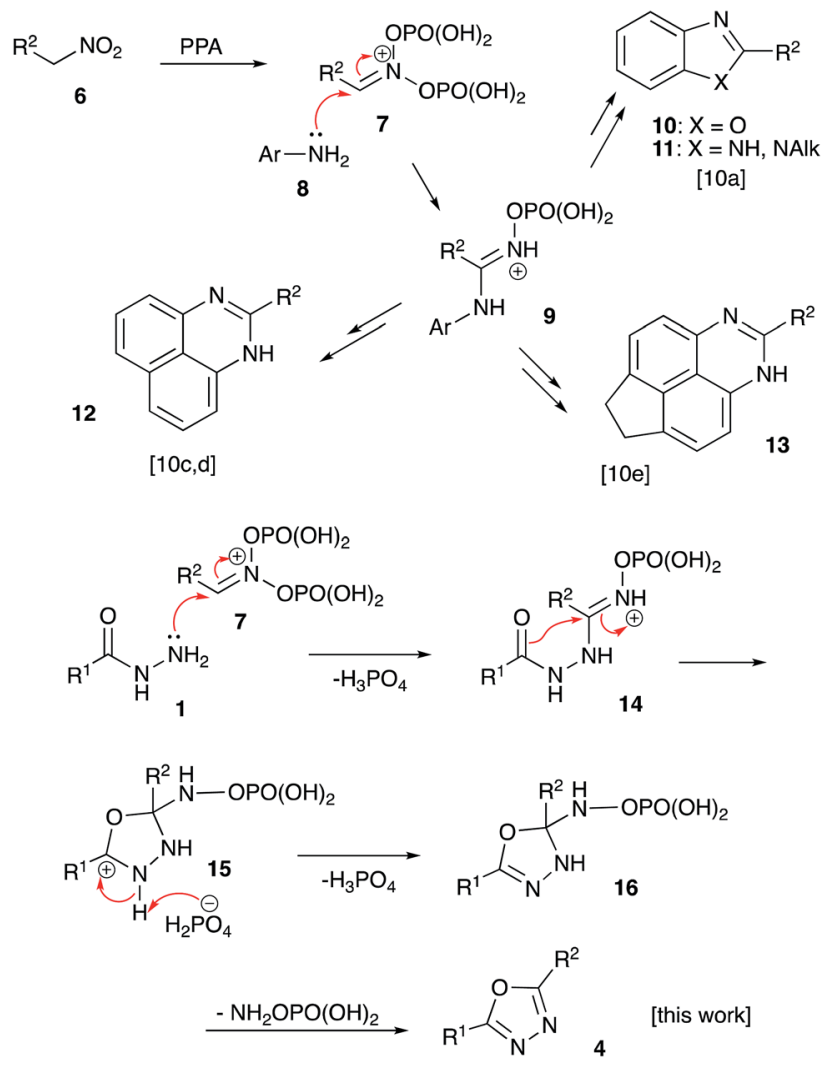

Scheme 2 intramolecular 5-exo-trig cyclization involving the carbonyl group of the hydrazide function and providing 4,5-dihydro1,3,4-oxadiazol-3-ium ion 15. The latter after deprotonation is expected to form 2,3-dihydro-1,3,4-oxadiazole 16, which after the elimination of $O$-phosphorylated hydroxylamine would provide desired 1,3,4-oxadizole 4 (Scheme 2).

To test this idea, a mixture benzohydrazide $\left(\mathbf{1 a}, \mathrm{R}^{1}=\mathrm{Ph}\right)$ and nitroethane $\left(6 \mathrm{a}, \mathrm{R}^{2}=\mathrm{Me}, 2.00\right.$ equiv.) was stirred in polyphosphoric acid $\left(86 \% \mathrm{P}_{2} \mathrm{O}_{5}\right)$ at $70{ }^{\circ} \mathrm{C}$ (Table 1, entry 1). However, even after 90 min no reaction was observed. At $90{ }^{\circ} \mathrm{C}$ the reaction proceeded slowly and after $30 \mathrm{~min}$ the conversion reached $14 \%$, however the main product of the reaction was 2,5diphenyl-1,3,4-oxadiazole $\left(\mathbf{5 a}, \mathrm{R}^{2}=\mathrm{Ph}\right)$. At $110{ }^{\circ} \mathrm{C}$ (boiling point of nitroethane) the process proceeded to completion affording mixtures of $10 \%$ of non-symmetric product 4 aa and $55 \%$ symmetric oxadiazole 5a (entry 3). The rest of the starting material polymerized forming highly polar resins. Employment of nitroethane (6a) in excess (3.00 equiv.) did not notably improve the reaction outcome (entries 4 and 5). Evidently, the symmetric product (5a) was formed via acid-mediated transacylation of 1a to produce intermediate symmetric $N, N^{\prime}$-diacylhydrazide $3 \mathbf{a}\left(\mathrm{R}^{2}=\mathrm{Ph}\right)$. In order to suppress this undesired process, we decided to add starting hydrazide 1a in small portions over an extended period of time. This resulted in only marginal improvement, however, in the reactions carried out in the presence of stoichiometric amount of nitroethane (entry 6). Gradual increase of the concentration of nitroethane (entries 710) resulted in both the conversion and chemoselectivity of the process to achieve exclusive formation of product $5 \mathbf{a}$ at $99 \%$ yield (entry 10). We also tested the possibility of employing Eaton's reagent $\left(\mathrm{P}_{2} \mathrm{O}_{5}\right.$ in $\left.\mathrm{MeSO}_{3} \mathrm{H}\right)$ as a mixture that is often used as an alternative to PPA in synthesis, but in this medium hydrazide 6a underwent complete hydrolysis to afford benzoic acid as the only detectible product of the reaction (entries 11 and 12).

With the optimized reaction conditions in hand we carried out the reaction between 1a and $\mathbf{6 a}$ in preparative

Table 1 Optimization of reaction conditions for cyclocondensation of $1 \mathrm{a}$ and $6 \mathrm{a}$ to produce oxadiazoles $4 \mathrm{aa}$ and $5 \mathrm{a}$

\begin{tabular}{lllll}
\hline & 6a (equiv.) & Medium & $T,{ }^{\circ} \mathrm{C}($ time) & $\mathbf{4 a a}^{\mathbf{5}} \mathbf{5} \mathbf{a}^{a}$ \\
\hline 1 & 1.00 & 86\% PPA & $70(1.5 \mathrm{~h})^{b}$ & $0: 0$ \\
2 & 1.00 & & $90(0.5 \mathrm{~h})^{b}$ & $0: 14$ \\
3 & 1.00 & & $110(0.5 \mathrm{~h})^{b}$ & $10: 55$ \\
4 & 3.00 & & $90(0.5 \mathrm{~h})^{b}$ & $0: 17$ \\
5 & 3.00 & & $110(1 \mathrm{~h})^{b}$ & $31: 61$ \\
6 & 1.00 & & $110(1 \mathrm{~h})^{c}$ & $12: 38$ \\
7 & 2.00 & & $110(1 \mathrm{~h})^{c}$ & $63: 25$ \\
8 & 3.00 & & $110(1 \mathrm{~h})^{c}$ & $64: 15$ \\
9 & 5.00 & & $110(1.5 \mathrm{~h})^{c}$ & $94: 6$ \\
$\mathbf{1 0}$ & 7.00 & & $\mathbf{1 1 0}(\mathbf{1 . 5 h})^{c}$ & $\mathbf{9 9}: \mathbf{0}$ \\
11 & 3.00 & Eaton's reagent $1: 7$ & $110(1 \mathrm{~h})$ & $0: 0^{d}$ \\
12 & 3.00 & Eaton's reagent $1: 10$ & $110(1 \mathrm{~h})$ & $0: 0^{d}$
\end{tabular}

${ }^{a}$ NMR yields of compounds $4 \mathbf{4 a}$ and $5 \mathbf{a}$ are shown. ${ }^{b}$ Benzohydrazide was added in a single portion. ${ }^{c}$ Benzohydrazide was slowly added by small portions. ${ }^{d}$ Complete hydrolysis of benzohydrazide was observed, affording benzoic acid as sole product. 


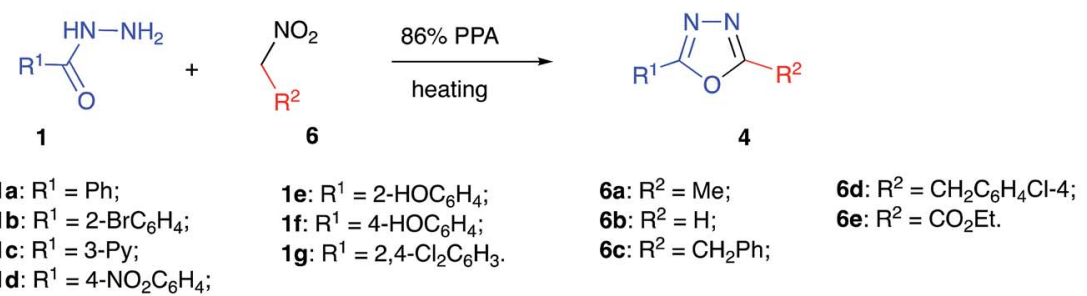<smiles>Cc1nnc(-c2ccccc2)o1</smiles>

(1) $4 \mathrm{aa}, 91 \%$

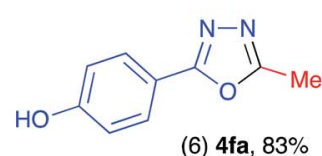

(6) $4 \mathrm{fa}, 83 \%$<smiles>Cc1nnc(-c2ccccc2Br)o1</smiles>

(2) $4 \mathrm{ba}, 76 \%$<smiles>Cc1nnc(-c2cccnc2)o1</smiles>

(3) $4 \mathrm{ca}, 99 \%$<smiles>Cc1nnc(-c2ccc([N+](=O)[O-])cc2)o1</smiles>

(4) $4 \mathrm{da}, 93 \%$<smiles>Cc1nnc(-c2ccccc2O)o1</smiles>

(5) $4 \mathrm{ea}, 87 \%$<smiles>Cc1nnc(-c2ccc(Cl)cc2Cl)o1</smiles>

(7) $4 \mathrm{ga}, 87 \%$

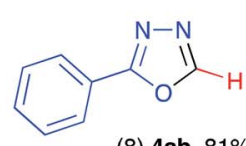

(8) $4 a b, 81 \%$

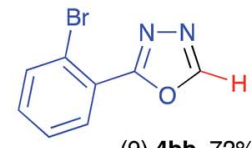

(9) $4 \mathbf{b b}, 72 \%$<smiles>O=[W]OCc1cccnc1</smiles>

(10) $4 \mathrm{cb}, 79 \%$<smiles>CC(C)(C)[14CH3]</smiles>

(11) $4 \mathrm{db}, 86 \%$<smiles>CC(C)(C)OCc1nnc(-c2ccccc2)o1</smiles><smiles>Oc1ccccc1-c1nnco1</smiles>

(12) $4 \mathrm{eb}, 81 \%$<smiles>Clc1ccc(Cc2nnc(-c3cccnc3)o2)cc1</smiles>

(17) $4 \mathrm{~cd}, 60 \%$

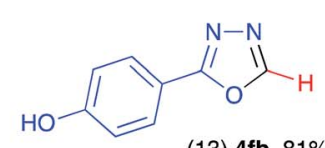

(13) $4 \mathrm{fb}, 81 \%$<smiles>CCOC(=O)c1nnc(-c2ccccc2)o1</smiles>

(18) $4 \mathrm{ae}, 64 \%$<smiles>Clc1ccc(-c2nnco2)c(Cl)c1</smiles>

(14) $4 \mathrm{gb}, 89 \%$<smiles>CCOC(=O)c1nnc(-c2ccccc2Br)o1</smiles>

(19) 4 be, $69 \%$<smiles>c1ccc(Cc2nnc(-c3ccccc3)o2)cc1</smiles>

(15) $4 a c, 59 \%$

(20) 4 ee, $70 \%$

Scheme 3

scale $(1.00 \mathrm{mmol})$. We were pleased to find, that oxadiazole 4aa was obtained in this reaction as sole product and isolated in high yield (Scheme 3, entry 1). Hydrazides 1b-g, derived from other aryl- and hetarylcarboxylic acids, also reacted with nitroethane (6a) smoothly, affording the corresponding heterocyclic products (entries 2-7). Remarkably, nonprotected phenols 1e and 1f also reacted uneventfully, which showcases the dual role of polyphosphoric acid as a versatile reaction medium with acidic properties and as a reagent for the reversible installation of temporary protecting phosphatyl groups.

Thorough analysis of literature revealed that synthetic access to monosubstituted oxadiazoles is especially challenging, since preparation of non-symmetric bis-hydrazides 2 required for this cyclo-condensation is severely complicated by relatively high strength formic acid and elevated electrophilicity of formohydrazide derivatives. ${ }^{13}$ This problem can be typically circumvented by usage of orthoformates as acylating agents. ${ }^{14} \mathrm{We}$ felt, however, that in our featured methodology, there should not be a dramatic difference between reactivity of nitroethane (6a) and nitromethane (6b). This would allow for the development of a very general synthetic protocol to access 1,3,4-oxadiazoles with different substitution patterns. To test this possibility, we carried out reactions with a series of benzohydrazides $(\mathbf{1 a}-\mathbf{g})$ in the presence of excess nitromethane $(\mathbf{6 b})$ at
$100{ }^{\circ} \mathrm{C}$ (boiling point of nitromethane). Gratifyingly, these reactions proceeded smoothly affording the corresponding 2aryl-1,3,4-oxadiazoles $\mathbf{4 a b} \mathbf{- 4 g b}$ in high yields (Scheme 3, entries 8-14). Along the same lines, we tested the possibility of employing (2-nitroethyl)benzenes (6c-d) as electrophilic components in the featured transformation. These reactions were more sluggish, probably due to excessive steric hindrance in the phosphorylated nitronate intermediates 7. It was found, however, that the cyclo-condensation could be pushed to complete conversion at $140{ }^{\circ} \mathrm{C}$ to afford the corresponding 5benzyloxadiazoles 4ac, 4ad, and 4cd albeit in somewhat lower yields (entries 15-17).

Another major challenge in chemistry of 1,3,4-oxadiazoles is associated with incorporation of ester substituents at C-2 of this heterocyclic scaffold. ${ }^{15}$ To address this issue we attempted the reaction of various benzohydrazides $(\mathbf{1 a}, \mathbf{b}, \mathbf{e})$ with ethyl 2-nitroacetate. We were pleased to discover that this reaction also proceeded smoothly under standard reaction conditions (temperature was raised to $130{ }^{\circ} \mathrm{C}$ to ensure complete conversion) affording the corresponding 1,3,4-oxadiazole-2carboxylates as the sole isolable product (Scheme 3, entries 18-20).

The formation and structural identity of compounds 5a, 4ga, and 4ca was unambiguously confirmed by a single crystal X-ray crystallography (Fig. 2). 

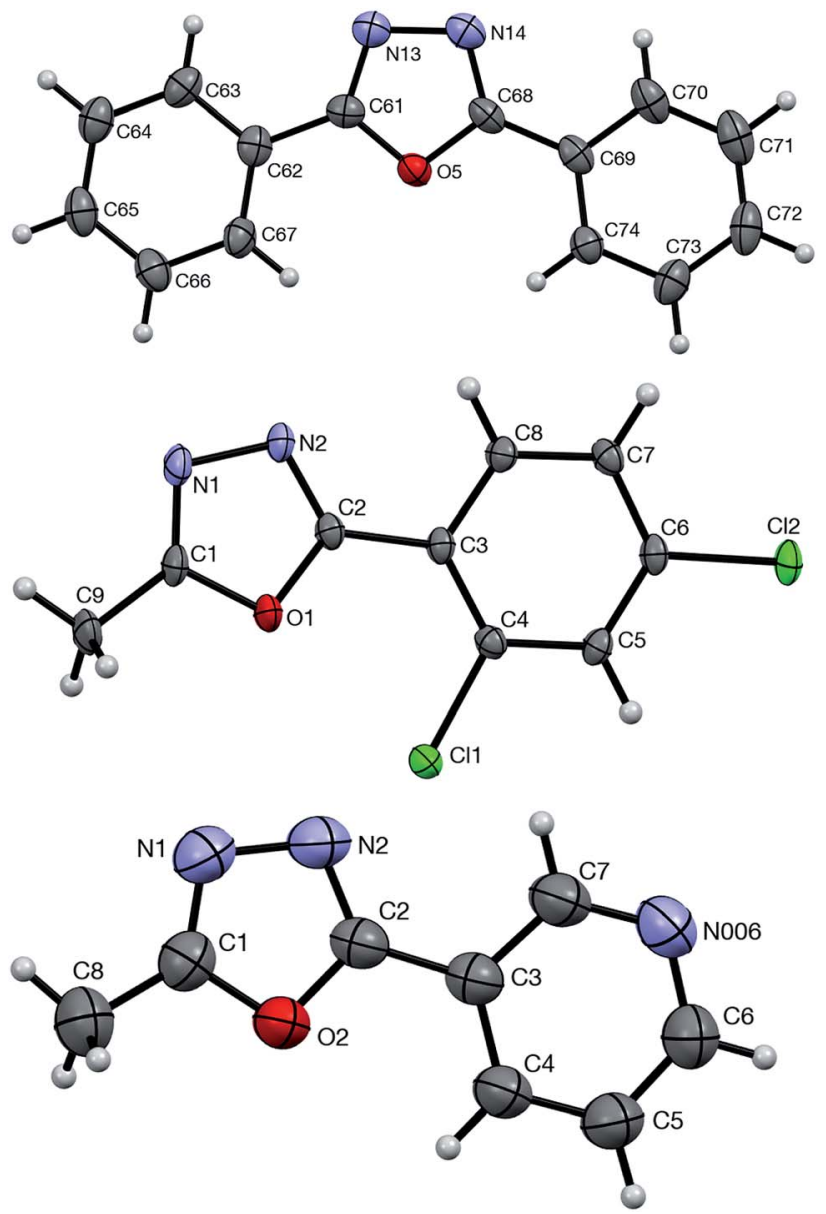

Fig. 2 ORTEP drawings of 5a (top, CCDC \#1875720), 4ga (middle, CCDC \#1875721), and 4ca (bottom, CCDC \#1875723) showing atom numbering schemes and $50 \%$ probability ellipsoids.

\section{Conclusion}

In conclusion, the novel one-pot multistep reaction sequence involving cyclo-condensation of acylhydrazides with electrophilicity-activated nitroalkanes was investigated. Careful optimization of the reaction conditions resulted in excellent chemo-selectivity of this newly developed synthetic protocol towards monosubstituted and non-symmetrically disubstituted 1,3,4-oxadiazoles. High yields of the resulting heterocyclic products and great functional group compatibility of this synthetic methodology was demonstrated.

\section{Experimental part}

\section{General information}

${ }^{1} \mathrm{H}$ and ${ }^{13} \mathrm{C}$ NMR spectra were recorded on a Bruker Avance-III spectrometer (400 or $100 \mathrm{MHz}$, respectively) equipped with a $\mathrm{BBO}$ probe in $\mathrm{CDCl}_{3}$ or DMSO- $d_{6}$, using TMS as an internal standard. High-resolution mass spectra were registered with a Bruker Maxis spectrometer (electrospray ionization, in MeCN solution, using $\mathrm{HCO}_{2} \mathrm{Na}-\mathrm{HCO}_{2} \mathrm{H}$ for calibration). Melting points were measured with a Stuart smp30 apparatus. All reactions were performed in oven-dried $5 \mathrm{~mL}$ round-bottomed flasks open to the atmosphere, employing overhead stirring. The reaction progress and purity of isolated compounds were controlled by TLC on Silufol UV-254 plates, with EtOAc as eluent. PPA was obtained by dissolving of $\mathrm{P}_{2} \mathrm{O}_{5}$ in $85 \%$ orthophosphoric acid according to the published protocol. ${ }^{16}$ All reagents and solvents were purchased from commercial vendors and used as received. Physical and spectral properties of compound $5 \mathbf{a}$ were identical to those reported in literature. ${ }^{17}$

2-Methyl-5-phenyl-1,3,4-oxadiazole (4aa V210). Typical procedure. A $10 \mathrm{~mL}$ Erlenmeyer flask equipped with magnetic bar and reflux condenser was charged with polyphosphoric acid $\left(86 \% \mathrm{P}_{2} \mathrm{O}_{5}, 1.5 \mathrm{~g}\right)$ and nitroethane (6a) $(500 \mu \mathrm{L}, 525 \mathrm{mg}, 7.00$ $\mathrm{mmol}$ ) and heated while stirring at $105-115^{\circ} \mathrm{C}$. Benzohydrazide (1a) $(136 \mathrm{mg}, 1.00 \mathrm{mmol})$ was added slowly in small portions over $1 \mathrm{~h}$, and the mixture was heated for an additional $30 \mathrm{~min}$, when TLC analysis showed the completion of the reaction. The mixture was diluted with water $(5 \mathrm{~mL})$, neutralized with $25 \%$ aqueous ammonia (4.5-5.0 mL is typically required), and extracted with EtOAc $(4 \times 5 \mathrm{~mL})$. Combined organic phases were concentrated and the crude product was purified by preparative column chromatography eluting with EtOAc/ petroleum ether mixture $1: 1$ to obtain the titled compound as a colorless solid, mp $63-65{ }^{\circ} \mathrm{C}$ (EtOAc), $R_{\mathrm{f}} 0.39$ (EtOAc/ petroleum ether, $1: 1)$. Yield $146 \mathrm{mg}(0.91 \mathrm{mmol}, 91 \%) .{ }^{1} \mathrm{H}$ NMR (400 MHz, $\left.\mathrm{CDCl}_{3}\right) \delta 8.02(\mathrm{~d}, J=7.8 \mathrm{~Hz}, 2 \mathrm{H}), 7.55-7.46(\mathrm{~m}$, 3H), 2.61 (s, 3H); ${ }^{13} \mathrm{C}$ NMR (101 MHz, $\left.\mathrm{CDCl}_{3}\right) \delta$ 165.0, 163.8, 131.7, 129.1, 126.8, 124.0, 11.3; IR (KBr, film, $\mathrm{cm}^{-1}$ ): 3060, 2943, 1582, 1556, 1487, 1450, 1252, 1095, 1073, 1021, 959; HRMS (ESI TOF) calculated for $\mathrm{C}_{9} \mathrm{H}_{8} \mathrm{~N}_{2} \mathrm{NaO}(\mathrm{M}+\mathrm{Na})^{+} 183.0529$, found 183.0531 (1.3 ppm).

2-(2-Bromophenyl)-5-methyl-1,3,4-oxadiazole (4ba V226). This material was obtained from 2-bromobenzohydrazide (1b) $(215 \mathrm{mg}, 1.00 \mathrm{mmol})$ and nitroethane $(6 \mathbf{a})(500 \mu \mathrm{L}, 525 \mathrm{mg}, 7.00$ $\mathrm{mmol})$. The reaction was carried at $105-115{ }^{\circ} \mathrm{C}$, and the titled compound was isolated as a yellowish oil, $R_{\mathrm{f}} 0.37$ (EtOAc/ petroleum ether, $1: 3)$. Yield $199 \mathrm{mg}(83 \%) .{ }^{1} \mathrm{H}$ NMR (400 $\left.\mathrm{MHz}, \mathrm{CDCl}_{3}\right) \delta 7.89(\mathrm{~d}, J=7.7 \mathrm{~Hz}, 1 \mathrm{H}), 7.74(\mathrm{~d}, J=8.0 \mathrm{~Hz}, 1 \mathrm{H})$, $7.44(\mathrm{t}, J=7.6 \mathrm{~Hz}, 1 \mathrm{H}), 7.38(\mathrm{t}, J=7.7 \mathrm{~Hz}, 1 \mathrm{H}), 2.64(\mathrm{~s}, 3 \mathrm{H}) ;{ }^{13} \mathrm{C}$ $\operatorname{NMR}\left(101 \mathrm{MHz}, \mathrm{CDCl}_{3}\right) \delta 164.3,164.0,134.6,132.5,131.7,127.7$, 125.5, 121.7, 11.3; IR (KBr, film, $\mathrm{cm}^{-1}$ ) 3071, 2932, 1586, 1435, 1347, 1241, 1098, 1021, 959; HRMS (ESI TOF) calculated for $\mathrm{C}_{9} \mathrm{H}_{7} \mathrm{BrN}_{2} \mathrm{NaO}(\mathrm{M}+\mathrm{Na})^{+} 260.9634$, found 260.9630 (1.7 ppm).

2-Methyl-5-(pyridin-3-yl)-1,3,4-oxadiazole (4ca V229). This material was obtained from nicotinohydrazide (1c) $(137 \mathrm{mg}$, $1.00 \mathrm{mmol})$ and nitroethane (6a) $(500 \mu \mathrm{L}, 525 \mathrm{mg}, 7.00 \mathrm{mmol})$. The reaction was carried out at $105-115{ }^{\circ} \mathrm{C}$, and the titled compound was isolated as a colorless solid, precipitated after aqueous work up and basification. No chromatographic purification was required. Mp $114-116{ }^{\circ} \mathrm{C}$ (EtOAc), $R_{\mathrm{f}} 0.43$ (EtOAc/ EtOH, $4: 1)$. Yield $159 \mathrm{mg}$ (0.99 mmol, 99\%). ${ }^{1} \mathrm{H}$ NMR (400 $\left.\mathrm{MHz}, \mathrm{CDCl}_{3}\right) \delta 9.25(\mathrm{~s}, 1 \mathrm{H}), 8.77(\mathrm{~d}, J=4.7 \mathrm{~Hz}, 1 \mathrm{H}), 8.38(\mathrm{~d}, J=$ $8.0 \mathrm{~Hz}, 1 \mathrm{H}), 7.50(\mathrm{dd}, J=7.9,5.0 \mathrm{~Hz}, 1 \mathrm{H}), 2.65(\mathrm{~s}, 3 \mathrm{H}) ;{ }^{13} \mathrm{C} \mathrm{NMR}$ $\left(101 \mathrm{MHz}, \mathrm{CDCl}_{3}\right) \delta 164.5,162.7,151.8,147.2,134.7,124.2$, 120.9, 11.9; IR (KBr, film, $\mathrm{cm}^{-1}$ ) 3082, 3049, 2932, 1593, 1575, 1549, 1468, 1432, 1351, 1259, 1087, 1010, 988, 959; HRMS (ESI TOF) calculated for $\mathrm{C}_{8} \mathrm{H}_{7} \mathrm{~N}_{3} \mathrm{NaO}(\mathrm{M}+\mathrm{Na})^{+} 184.0481$, found $183.0482(0.2 \mathrm{ppm})$. 
2-Methyl-5-(4-nitrophenyl)-1,3,4-oxadiazole (4da V233). This material was obtained from 4-nitrobenzohydrazide (1d) (181 mg, $1.00 \mathrm{mmol}$ ) and nitroethane (6a) $(500 \mu \mathrm{L}, 525 \mathrm{mg}, 7.00$ $\mathrm{mmol}$ ). The reaction was carried out at $105-115{ }^{\circ} \mathrm{C}$, and the titled compound was isolated as a yellow solid, mp $168-170{ }^{\circ} \mathrm{C}$ (EtOAc), $R_{\mathrm{f}} 0.27$ (EtOAc/petroleum ether, $\left.1: 1\right)$. Yield $191 \mathrm{mg}$ (0.93 mmol, 93\%). ${ }^{1} \mathrm{H}$ NMR (400 $\left.\mathrm{MHz}, \mathrm{CDCl}_{3}\right) \delta 8.37$ (d, $J=$ $8.7 \mathrm{~Hz}, 2 \mathrm{H}), 8.22(\mathrm{~d}, J=8.6 \mathrm{~Hz}, 2 \mathrm{H}), 2.67(\mathrm{~s}, 3 \mathrm{H}) ;{ }^{13} \mathrm{C}$ NMR $(101$ $\left.\mathrm{MHz}, \mathrm{CDCl}_{3}\right) \delta 164.9,163.4,149.6,129.6,127.8,124.5,11.3$; IR (KBr, film, $\mathrm{cm}^{-1}$ ) 3108, 3071, 3016, 2932, 1578, 1553, 1520, 1351, 1333, 1311, 1292, 1234, 1087, 1032, 867; HRMS (ESI TOF) calculated for $\mathrm{C}_{9} \mathrm{H}_{7} \mathrm{~N}_{3} \mathrm{NaO}_{3}(\mathrm{M}+\mathrm{Na})^{+} 228.0380$, found 228.0378 (0.8 ppm).

2-(5-Methyl-1,3,4-oxadiazol-2-yl)phenol (4ea V235). This material was obtained from 2-hydroxybenzohydrazide (1e) (152 mg, $1.00 \mathrm{mmol}$ ) and nitroethane (6a) $(500 \mu \mathrm{L}, 525 \mathrm{mg}, 7.00$ $\mathrm{mmol})$. The reaction was carried out at $105-115{ }^{\circ} \mathrm{C}$, and the titled compound was isolated as a light-brown solid, mp 73$75{ }^{\circ} \mathrm{C}$ (acetone/EtOAc), $R_{\mathrm{f}} 0.38$ (EtOAc/petroleum ether, $1: 3$ ). Yield $143 \mathrm{mg}$ (0.87 mmol, 87\%). ${ }^{1} \mathrm{H}$ NMR (400 MHz, $\mathrm{CDCl}_{3}$ ) $\delta 10.12(\mathrm{br}, \mathrm{s}, 1 \mathrm{H}), 7.73(\mathrm{dd}, J=7.9,1.5 \mathrm{~Hz}, 1 \mathrm{H}), 7.47-7.38(\mathrm{~m}$, $1 \mathrm{H}), 7.12(\mathrm{~d}, J=8.4 \mathrm{~Hz}, 1 \mathrm{H}), 7.04-6.94(\mathrm{~m}, 1 \mathrm{H}), 2.65(\mathrm{~s}, 3 \mathrm{H}) ;{ }^{13} \mathrm{C}$ NMR (101 MHz, $\left.\mathrm{CDCl}_{3}\right) \delta$ 164.7, 162.6, 157.6, 133.6, 126.5, 120.0, 117.7, 108.3, 11.2; IR (KBr, film, $\mathrm{cm}^{-1}$ ) 3189, 3071, 2976, 2943, 1629, 1593, 1549, 1490, 1439, 1410, 1355, 1300, 1241, 1080, 1040, 963; HRMS (ESI TOF) calculated for $\mathrm{C}_{9} \mathrm{H}_{8} \mathrm{~N}_{2} \mathrm{NaO}_{2}(\mathrm{M}+$ $\mathrm{Na})^{+}$199.0478, found 199.0476, (1.0 ppm).

4-(5-Methyl-1,3,4-oxadiazol-2-yl)phenol (4fa V242). This material was obtained from 4-hydroxybenzohydrazide (1f) (152 mg, $1.00 \mathrm{mmol}$ ) and nitroethane (6a) (500 $\mu \mathrm{L}, 525 \mathrm{mg}, 7.00$ $\mathrm{mmol})$. The reaction was carried out at $105-115{ }^{\circ} \mathrm{C}$, and the titled compound was isolated as a light-brown solid, mp 236$238{ }^{\circ} \mathrm{C}$ (acetone/EtOAc); $R_{\mathrm{f}} 0.30$ (EtOAc/petroleum ether, $2: 1$ ). Yield $146 \mathrm{mg}(0.83 \mathrm{mmol}, 83 \%) .{ }^{1} \mathrm{H}$ NMR (400 MHz, DMSO- $d_{6}$ ) $\delta 10.28(\mathrm{~s}, 1 \mathrm{H}), 7.79$ (d, $J=8.7 \mathrm{~Hz}, 2 \mathrm{H}), 6.93(\mathrm{~d}, J=8.7 \mathrm{~Hz}, 2 \mathrm{H})$, $2.53(\mathrm{~s}, 3 \mathrm{H}) ;{ }^{13} \mathrm{C}$ NMR (101 MHz, DMSO- $\left.d_{6}\right) \delta 164.1,163.1,160.6$, 128.3, 116.2, 114.4, 10.6. IR (KBr, film, $\mathrm{cm}^{-1}$ ) 3156, 3020, 2947, 1611, 1589, 1494, 1377, 1289, 1234, 1164, 1120, 1073, 933, 853; HRMS (ESI TOF) calculated for $\mathrm{C}_{9} \mathrm{H}_{8} \mathrm{~N}_{2} \mathrm{NaO}_{2}(\mathrm{M}+\mathrm{Na})^{+} 199.0478$, found 199.0482 (1.9 ppm).

2-(2,4-Dichlorophenyl)-5-methyl-1,3,4-oxadiazole (4ga V240). This material was obtained from 2,4-dichlorobenzohydrazide (1g) $(205 \mathrm{mg}, 1.00 \mathrm{mmol})$ and nitroethane (6a) $(500 \mu \mathrm{L}, 525 \mathrm{mg}$, $7.00 \mathrm{mmol})$. The reaction was carried out at $105-115^{\circ} \mathrm{C}$, and the titled compound was isolated as a colorless solid, mp $92-102{ }^{\circ} \mathrm{C}$ (EtOAc), $R_{\mathrm{f}} 0.50$ (EtOAc/petroleum ether, $1: 1$ ). Yield $199 \mathrm{mg}$ (0.87 mmol, 87\%). ${ }^{1} \mathrm{H}$ NMR (400 $\left.\mathrm{MHz}, \mathrm{CDCl}_{3}\right) \delta 7.92$ (d, $J=$ $8.5 \mathrm{~Hz}, 1 \mathrm{H}), 7.56(\mathrm{~d}, J=1.9 \mathrm{~Hz}, 1 \mathrm{H}), 7.39(\mathrm{dd}, J=8.5,1.9 \mathrm{~Hz}$, 1H), 2.64 (s, 3H); ${ }^{13} \mathrm{C}$ NMR (101 MHz, $\left.\mathrm{CDCl}_{3}\right) \delta$ 164.4, 162.7, 138.1, 133.9, 131.9, 131.3, 127.7, 121.9, 11.3; IR (KBr, film, $\mathrm{cm}^{-1}$ ) 3104, 3086, 3009, 2936, 1589, 1564, 1454, 1417, 1270, 1241, 1106, 1018, 963, 879; HRMS (ESI TOF) calculated for $\mathrm{C}_{9} \mathrm{H}_{6} \mathrm{Cl}_{2}$ $\mathrm{N}_{2} \mathrm{NaO}(\mathrm{M}+\mathrm{Na})^{+} 250.9749$, found 250.9747 (0.8 ppm).

2-Phenyl-1,3,4-oxadiazole (4ab V216). This material was obtained from benzohydrazide (1a) $(136 \mathrm{mg}, 1.00 \mathrm{mmol})$ and nitromethane $(6 \mathbf{b})(581 \mu \mathrm{L}, 610 \mathrm{mg}, 10.0 \mathrm{mmol})$. The reaction was carried out at $95-105{ }^{\circ} \mathrm{C}$, and the titled compound was isolated as a colorless oil, $R_{\mathrm{f}} 0.24$ (EtOAc/petroleum ether, $1: 3$ ). Yield $119 \mathrm{mg}$ (0.81 mmol, 81\%). ${ }^{1} \mathrm{H}$ NMR (400 $\mathrm{MHz}, \mathrm{CDCl}_{3}$ ) $\delta 8.48(\mathrm{~s}, 1 \mathrm{H}), 8.11-8.05(\mathrm{~m}, 2 \mathrm{H}), 7.57-7.49(\mathrm{~m}, 3 \mathrm{H}) ;{ }^{13} \mathrm{C} \mathrm{NMR}$ $\left(101 \mathrm{MHz}, \mathrm{CDCl}_{3}\right) \delta 164.9,152.8,132.2,129.3,127.2,123.6$; IR (KBr, film, $\mathrm{cm}^{-1}$ ) 3064, 1578, 1556, 1487, 1450, 1355, 1248, 1091, 1076, 1025, 959, 930; HRMS (ESI TOF) calculated for $\mathrm{C}_{8} \mathrm{H}_{6} \mathrm{~N}_{2} \mathrm{NaO}(\mathrm{M}+\mathrm{Na})^{+}$169.0372, found 169.0373 (0.3 ppm).

2-(2-Bromophenyl)-1,3,4-oxadiazole (4bb V232). This material was obtained from 2-bromobenzohydrazide (1b) $215 \mathrm{mg}$, $1.00 \mathrm{mmol}$ ) and nitromethane (6b) $(581 \mu \mathrm{L}, 610 \mathrm{mg}, 10.0 \mathrm{mmol})$. The reaction was carried out at $95-105{ }^{\circ} \mathrm{C}$, and the titled compound was isolated as a yellow oil, $R_{\mathrm{f}} 0.28$ (EtOAc/petroleum ether, $1: 3)$. Yield $162 \mathrm{mg}(0.72 \mathrm{mmol}, 72 \%) .{ }^{1} \mathrm{H}$ NMR $(400 \mathrm{MHz}$, $\left.\mathrm{CDCl}_{3}\right) \delta 8.57(\mathrm{~s}, 1 \mathrm{H}), 7.94(\mathrm{~d}, J=7.7 \mathrm{~Hz}, 1 \mathrm{H}), 7.77(\mathrm{~d}, J=7.9 \mathrm{~Hz}$, $1 \mathrm{H}), 7.48(\mathrm{t}, J=7.5 \mathrm{~Hz}, 1 \mathrm{H}), 7.41(\mathrm{t}, J=7.7 \mathrm{~Hz}, 1 \mathrm{H}) ;{ }^{13} \mathrm{C} \mathrm{NMR}$ $\left(101 \mathrm{MHz} \mathrm{CDCl}_{3}\right) \delta 163.9,153.2,134.7,132.9,131.9,127.8$, 125.1, 121.9; IR (KBr, film, $\mathrm{cm}^{-1}$ ) 3119, 3002, 1597, 1575, 1516, 1457, 1432, 1234, 1109, 1084, 1025, 952; HRMS (ESI TOF) calculated for $\mathrm{C}_{8} \mathrm{H}_{5} \mathrm{BrN}_{2} \mathrm{NaO}(\mathrm{M}+\mathrm{Na})^{+} 246.9477$, found 246.9479 (0.6 ppm).

2-(Pyridin-3-yl)-1,3,4-oxadiazole (4cb V230). This material was obtained from nicotinohydrazide (1c) $(137 \mathrm{mg}, 1.00 \mathrm{mmol})$ and nitromethane $(6 \mathbf{b})(581 \mu \mathrm{L}, 610 \mathrm{mg}, 10.0 \mathrm{mmol})$. The reaction was carried out at $95-105^{\circ} \mathrm{C}$, and the titled compound was isolated after extraction with dichloromethane $(4 \times 5 \mathrm{~mL})$ as a colorless solid, mp 72-73 ${ }^{\circ} \mathrm{C}$ (EtOAc); $R_{\mathrm{f}} 0.51$ (EtOAc/EtOH, $4:$ 1). Yield $116 \mathrm{mg}$ (0.79 mmol, 79\%). ${ }^{1} \mathrm{H}$ NMR $(400 \mathrm{MHz}$, $\left.\mathrm{CDCl}_{3}\right) \delta 9.31(\mathrm{~s}, 1 \mathrm{H}), 8.84-8.76(\mathrm{~m}, 1 \mathrm{H}), 8.55(\mathrm{~s}, 1 \mathrm{H}), 8.39(\mathrm{~d}, J=$ $8.0 \mathrm{~Hz}, 1 \mathrm{H}), 7.49$ (dd, $J=8.0,4.9 \mathrm{~Hz}, 1 \mathrm{H}) ;{ }^{13} \mathrm{C}$ NMR $(101 \mathrm{MHz}$, $\left.\mathrm{CDCl}_{3}\right) \delta 162.9,153.2,152.9,148.2,134.6,124.0,120.2,77.2$; IR (KBr, film, $\mathrm{cm}^{-1}$ ) 3332, 3071, 2998, 1608, 1586, 1556, 1512, 1468, 1432, 1267, 1131, 1102, 1073, 1021, 952, 897; HRMS (ESI TOF) calculated for $\mathrm{C}_{7} \mathrm{H}_{5} \mathrm{~N}_{3} \mathrm{NaO}(\mathrm{M}+\mathrm{Na})^{+} 170.0325$, found 170.0330 (3.1 ppm).

2-(4-Nitrophenyl)-1,3,4-oxadiazole (4db V245). This material was obtained from 4-nitrobenzohydrazide (1d) $(181 \mathrm{mg}, 1.00$ $\mathrm{mmol})$ and nitromethane $(\mathbf{6 b})(581 \mu \mathrm{L}, 610 \mathrm{mg}, 10.0 \mathrm{mmol})$. The reaction was carried out at $95-105{ }^{\circ} \mathrm{C}$, and the titled compound was isolated as a yellow solid, $\mathrm{mp} 152-153{ }^{\circ} \mathrm{C}$ (EtOAc), $R_{\mathrm{f}} 0.32$ (EtOAc/petroleum ether, 1 : 2). Yield $165 \mathrm{mg}$ (0.86 mmol, 86\%). ${ }^{1} \mathrm{H}$ NMR $\left(400 \mathrm{MHz}, \mathrm{CDCl}_{3}\right) \delta 8.58(\mathrm{~s}, 1 \mathrm{H}), 8.40(\mathrm{~d}, J=8.9 \mathrm{~Hz}, 2 \mathrm{H})$, $8.30(\mathrm{~d}, J=8.9 \mathrm{~Hz}, 2 \mathrm{H}) ;{ }^{13} \mathrm{C} \mathrm{NMR}\left(101 \mathrm{MHz}, \mathrm{CDCl}_{3}\right) \delta 163.3$, 153.6, 149.9, 129.1, 128.3, 124.6; IR (KBr, film, $\mathrm{cm}^{-1}$ ) 3163, 3108, 3075, 3009, 1611, 1560, 1520, 1333, 1311, 1296, 1113, 1065, 948, 853; HRMS (ESI TOF) calculated for $\mathrm{C}_{8} \mathrm{H}_{5} \mathrm{~N}_{3} \mathrm{NaO}_{3}(\mathrm{M}+\mathrm{Na})^{+}$ 214.0223, found 214.0225 (1.1 ppm).

2-(1,3,4-Oxadiazol-2-yl)phenol (4eb V247). This material was obtained from 2-hydroxybenzohydrazide (1e) (152 mg, 1.00 $\mathrm{mmol})$ and nitromethane $(6 \mathrm{~b})(581 \mu \mathrm{L}, 610 \mathrm{mg}, 10.0 \mathrm{mmol})$. The reaction was carried out at $95-105{ }^{\circ} \mathrm{C}$, and the titled compound was isolated as a colorless solid, $\mathrm{mp} 84-86{ }^{\circ} \mathrm{C}$ (acetone), $R_{\mathrm{f}} 0.35$ (EtOAc/petroleum ether, $1: 3)$. Yield $131 \mathrm{mg}$ (0.81 mmol, 81\%); ${ }^{1} \mathrm{H}$ NMR $\left(400 \mathrm{MHz}, \mathrm{CDCl}_{3}\right) \delta 10.06(\mathrm{~s}, 1 \mathrm{H}), 8.47(\mathrm{~s}, 1 \mathrm{H}), 7.79(\mathrm{~d}, J$ $=7.8 \mathrm{~Hz}, 1 \mathrm{H}), 7.47(\mathrm{t}, J=7.7 \mathrm{~Hz}, 1 \mathrm{H}), 7.14(\mathrm{~d}, J=8.4 \mathrm{~Hz}, 1 \mathrm{H})$, $7.02(\mathrm{t}, J=7.5 \mathrm{~Hz}, 1 \mathrm{H}) ;{ }^{13} \mathrm{C} \mathrm{NMR}\left(101 \mathrm{MHz}, \mathrm{CDCl}_{3}\right) \delta 164.5$, $157.8,151.5,134.2,126.9,120.2,117.8,107.9$; IR $(\mathrm{KBr}$, 
film, $\mathrm{cm}^{-1}$ ) 3222, 3148, 3068, 1769, 1728, 1626, 1586, 1549, 1490, 1406, 1303, 1256, 1157, 1095, 1058, 952; HRMS (ESI TOF) calculated for $\mathrm{C}_{8} \mathrm{H}_{6} \mathrm{~N}_{2} \mathrm{NaO}_{2}(\mathrm{M}+\mathrm{Na})^{+} 185.0321$, found 185.0320 (1.0 ppm).

4-(1,3,4-Oxadiazol-2-yl)phenol (4fb V260). This material was obtained from 4-hydroxybenzohydrazide (1f) (152 mg, 1.00 $\mathrm{mmol})$ and nitromethane $(6 \mathrm{~b})(581 \mu \mathrm{L}, 610 \mathrm{mg}, 10.0 \mathrm{mmol})$. The reaction was carried out at $95-105{ }^{\circ} \mathrm{C}$, and the titled compound was isolated as a colorless solid, mp 127-129 ${ }^{\circ} \mathrm{C}$ (acetone), $R_{\mathrm{f}}$ 0.34 (EtOAc/petroleum ether, $1: 1)$. Yield $130 \mathrm{mg}(0.81 \mathrm{mmol}$, 81\%). ${ }^{1} \mathrm{H}$ NMR (400 MHz, $\mathrm{CDCl}_{3}$ ) $\delta 10.33$ (s, 1H), $9.23(\mathrm{~s}, 1 \mathrm{H})$, 7.85 (d, $J=8.6 \mathrm{~Hz}, 2 \mathrm{H}), 6.95(\mathrm{~d}, J=8.6 \mathrm{~Hz}, 2 \mathrm{H}) ;{ }^{13} \mathrm{C} \mathrm{NMR}(101$ $\left.\mathrm{MHz}, \mathrm{CDCl}_{3}\right) \delta 163.8,160.9,153.8,128.7,116.2,114.0$; IR $(\mathrm{KBr}$, film, $\mathrm{cm}^{-1}$ ) 3147, 3027, 1615, 1597, 1498, 1384, 1285, 1245, 1179, 1128, 1065, 966, 941, 853; HRMS (ESI TOF) calculated for $\mathrm{C}_{8} \mathrm{H}_{6} \mathrm{~N}_{2} \mathrm{NaO}_{2}(\mathrm{M}+\mathrm{Na})^{+}:$185.0321, found 185.0326 (2.3 ppm).

2-(2,4-Dichlorophenyl)-1,3,4-oxadiazole (4gb V246). This material was obtained from 2,4-dichlorobenzohydrazide (1g) (205 $\mathrm{mg}, 1.00 \mathrm{mmol}$ ) and nitromethane (6b) $(581 \mu \mathrm{L}, 610 \mathrm{mg}$, $10.0 \mathrm{mmol})$. The reaction was carried out at $95-105^{\circ} \mathrm{C}$, and the titled compound was isolated as a colorless solid, mp 141$151{ }^{\circ} \mathrm{C}$ (EtOAc), $R_{\mathrm{f}} 0.41$ (EtOAc/petroleum ether, $1: 2$ ). Yield $192 \mathrm{mg}$ (0.89 mmol, 89\%). ${ }^{1} \mathrm{H}$ NMR (400 MHz, $\left.\mathrm{CDCl}_{3}\right) \delta 8.57$ (s, $1 \mathrm{H}), 7.96(\mathrm{~d}, J=8.5 \mathrm{~Hz}, 1 \mathrm{H}), 7.58(\mathrm{~d}, J=1.9 \mathrm{~Hz}, 1 \mathrm{H}), 7.41(\mathrm{dd}, J=$ 8.5, $1.8 \mathrm{~Hz}, 1 \mathrm{H}) ;{ }^{13} \mathrm{C} \mathrm{NMR}\left(101 \mathrm{MHz}, \mathrm{CDCl}_{3}\right) \delta 162.7,153.2$, 138.6, 134.2, 132.1, 131.4, 127.8, 121.4; IR (KBr, film, $\mathrm{cm}^{-1}$ ) 3314, 3204, 3093, 3027, 1659, 1633, 1593, 1512, 1457, 1377, 1307, 1252, 1106, 1058, 959, 893, 864; HRMS (ESI TOF) calculated for $\mathrm{C}_{8} \mathrm{H}_{4} \mathrm{Cl}_{2} \mathrm{~N}_{2} \mathrm{NaO}(\mathrm{M}+\mathrm{Na})^{+} 236.9593$, found 236.9588 (2.1 $\mathrm{ppm})$.

2-Benzyl-5-phenyl-1,3,4-oxadiazole (4ac V251). This material was obtained from benzohydrazide (1a) (136 mg, $1.00 \mathrm{mmol}$ ) and (2-nitroethyl)benzene (6c) $(202 \mu \mathrm{L}, 226 \mathrm{mg}, 1.50 \mathrm{mmol})$. The reaction was carried out at $130-135^{\circ} \mathrm{C}$, and the titled compound was isolated as a colorless solid, mp 93-95 ${ }^{\circ} \mathrm{C}$ (EtOAc), $R_{\mathrm{f}} 0.35$ (EtOAc/petroleum ether, 1 : 3). Yield $139 \mathrm{mg}$ (0.59 mmol, 59\%). ${ }^{1} \mathrm{H}$ NMR (400 MHz, $\left.\mathrm{CDCl}_{3}\right) \delta$ 8.04-7.97 (m, 2H), 7.52-7.45 (m, $3 \mathrm{H}), 7.36(\mathrm{~d}, J=4.3 \mathrm{~Hz}, 3 \mathrm{H}), 7.31(\mathrm{dd}, J=8.6,4.2 \mathrm{~Hz}, 1 \mathrm{H}), 4.29$ $(\mathrm{s}, 2 \mathrm{H}) ;{ }^{13} \mathrm{C}$ NMR $\left(101 \mathrm{MHz}, \mathrm{CDCl}_{3}\right) \delta$ 165.4, 165.3, 134.0, 131.8, 129.12 , 129.08, 129.0, 127.7, 127.0, 124.0, 32.1; IR (KBr, film, $\mathrm{cm}^{-1}$ ) 3075, 2928, 2858, 1567, 1553, 1490, 1454, 1424, 1256, 1095, 1065, 1010, 959; HRMS (ESI TOF) calculated for $\mathrm{C}_{15} \mathrm{H}_{12} \mathrm{~N}_{2} \mathrm{NaO}(\mathrm{M}+\mathrm{Na})^{+}$: 259.0842, found $259.0842(0.0 \mathrm{ppm})$.

2-(4-Chlorobenzyl)-5-phenyl-1,3,4-oxadiazole (4ad V253). This material was obtained from benzohydrazide (1a) $(136 \mathrm{mg}$, $1.00 \mathrm{mmol}$ ) and 1-chloro-4-(2-nitroethyl)benzene (6d) $(220 \mu \mathrm{L}$, $278 \mathrm{mg}, 1.50 \mathrm{mmol})$. The reaction was carried out at $130-135{ }^{\circ} \mathrm{C}$, and the titled compound was isolated as a colorless solid, $\mathrm{mp}$ $107-109{ }^{\circ} \mathrm{C}$ (EtOAc); $R_{\mathrm{f}} 0.24$ (EtOAc/petroleum ether, $1: 3$ ). Yield $162 \mathrm{mg}(0.60 \mathrm{mmol}, 60 \%) .{ }^{1} \mathrm{H}$ NMR $\left(400 \mathrm{MHz}, \mathrm{CDCl}_{3}\right) \delta 8.00$ (dd, $J=8.0,1.4 \mathrm{~Hz}, 2 \mathrm{H}), 7.53-7.45(\mathrm{~m}, 3 \mathrm{H}), 7.32(\mathrm{q}, J=8.6 \mathrm{~Hz}, 4 \mathrm{H})$, 4.25 (s, 2H); ${ }^{13} \mathrm{C}$ NMR (101 MHz, $\left.\mathrm{CDCl}_{3}\right) \delta 165.4,164.9,133.7$, 132.4, 131.9, 130.3, 129.3, 129.2, 127.0, 123.8, 31.4; IR (KBr, film, $\mathrm{cm}^{-1}$ ) 3068, 2928, 2855, 1553, 1490, 1450, 1380, 1194, 1087, 1010, 961; HRMS (ESI TOF) calculated for $\mathrm{C}_{15} \mathrm{H}_{11} \mathrm{ClN}_{2} \mathrm{NaO}$ $(\mathrm{M}+\mathrm{Na})^{+} 293.0452$, found 293.0460 (2.7 ppm).
2-(4-Chlorobenzyl)-5-(pyridin-3-yl)-1,3,4-oxadiazole

(4cd V254). This material was obtained from nicotinohydrazide (1c) (137 mg, $1.00 \mathrm{mmol}$ ) and 1-chloro-4-(2-nitroethyl)benzene (6c) $(220 \mu \mathrm{L}, 278 \mathrm{mg}, 1.50 \mathrm{mmol})$. The reaction was carried out at $130-135{ }^{\circ} \mathrm{C}$, and the titled compound was isolated as a colorless solid, mp 112-114 ${ }^{\circ} \mathrm{C}$ (EtOAc), $R_{\mathrm{f}} 0.34$ (EtOAc/petroleum ether, $1: 3)$. Yield $146 \mathrm{mg}$ (0.54 mmol, 54\%). ${ }^{1} \mathrm{H}$ NMR (400 MHz, $\left.\mathrm{CDCl}_{3}\right) \delta 9.22(\mathrm{~s}, 1 \mathrm{H}), 8.77(\mathrm{~d}, J=4.3 \mathrm{~Hz}, 1 \mathrm{H}), 8.35(\mathrm{~d}, J=7.9 \mathrm{~Hz}$, $1 \mathrm{H}), 7.49$ (dd, $J=7.8,4.9 \mathrm{~Hz}, 1 \mathrm{H}), 7.33$ (q, $J=8.5 \mathrm{~Hz}, 4 \mathrm{H}), 4.28$ $(\mathrm{s}, 2 \mathrm{H}) ;{ }^{13} \mathrm{C}$ NMR $\left(101 \mathrm{MHz}, \mathrm{CDCl}_{3}\right) \delta 165.7,163.1,151.8,147.2$, 134.9 , 134.0, 132.0, 130.4, 129.4, 124.2, 120.7, 31.4; IR (KBr, film, $\mathrm{cm}^{-1}$ ) 3090, 3064, 2958, 2925, 2855, 1567, 1490, 1413, 1256, 1186, 1084, 1007, 963, 853; HRMS calculated for $\mathrm{C}_{14} \mathrm{H}_{10^{-}}$ $\mathrm{ClN}_{3} \mathrm{NaO}(\mathrm{M}+\mathrm{Na})^{+}$294.0405, found 293.0395, (3.2 ppm).

Ethyl 5-phenyl-1,3,4-oxadiazole-2-carboxylate (4ae V269). This material was obtained from benzohydrazide (1a) $(136 \mathrm{mg}$, $1.00 \mathrm{mmol}$ ) and ethyl 2-nitroacetate (6e) $(165 \mu \mathrm{L}, 200 \mathrm{mg}, 1.50$ $\mathrm{mmol})$. The reaction was carried out at $120-130{ }^{\circ} \mathrm{C}$, and the titled compound was isolated as a colorless oil, $R_{\mathrm{f}} 0.47$ (EtOAc/ petroleum ether, $1: 3)$. Yield $136 \mathrm{mg}$ (0.64 mmol, 64\%). ${ }^{1} \mathrm{H}$ NMR $\left(400 \mathrm{MHz}, \mathrm{CDCl}_{3}\right) \delta 8.20-8.14(\mathrm{~m}, 2 \mathrm{H}), 7.63-7.58(\mathrm{~m}, 1 \mathrm{H}), 7.57-$ $7.52(\mathrm{~m}, 2 \mathrm{H}), 4.56(\mathrm{q}, J=7.1 \mathrm{~Hz}, 2 \mathrm{H}), 1.48(\mathrm{t}, J=7.1 \mathrm{~Hz}, 3 \mathrm{H}) ;{ }^{13} \mathrm{C}$ NMR (101 MHz, $\left.\mathrm{CDCl}_{3}\right) \delta 166.6,156.6,154.6,133.0,129.4,127.8$, 122.9, 63.7, 14.3; IR (KBr, film, $\mathrm{cm}^{-1}$ ) 3078, 2994, 2941, 1748, 1625, 1546, 1478, 1452, 1377, 1242, 1190, 1163, 1070, 1017, 841, 792, 713, 691; HRMS (ESI TOF) calculated for $\mathrm{C}_{11} \mathrm{H}_{10} \mathrm{~N}_{2} \mathrm{NaO}_{3}(\mathrm{M}$ $+\mathrm{Na})^{+} 241.0584$, found 241.0577 (2.8 ppm).

Ethyl 5-(2-bromophenyl)-1,3,4-oxadiazole-2-carboxylate (4be V273). This material was obtained from 2-bromobenzohydrazide (1b) (215 mg, $1.00 \mathrm{mmol})$ and ethyl 2-nitroacetate (6e) $(165 \mu \mathrm{L}$, $200 \mathrm{mg}, 1.50 \mathrm{mmol})$. The reaction was carried out at $120-130{ }^{\circ} \mathrm{C}$, and the titled compound was isolated as a colorless oil, $R_{\mathrm{f}} 0.30$ (EtOAc/petroleum ether, $1: 3$ ). Yield $205 \mathrm{mg}$ (0.69 mmol, 69\%). ${ }^{1} \mathrm{H}$ NMR (400 MHz, $\left.\mathrm{CDCl}_{3}\right) \delta 8.00(\mathrm{dd}, J=7.6,1.8 \mathrm{~Hz}, 1 \mathrm{H}), 7.79$ $(\mathrm{dd}, J=7.8,1.3 \mathrm{~Hz}, 1 \mathrm{H}), 7.49(\mathrm{td}, J=7.5,1.4 \mathrm{~Hz}, 1 \mathrm{H}), 7.44(\mathrm{td}, J=$ $7.7,1.9 \mathrm{~Hz}, 1 \mathrm{H}), 4.56$ (q, $J=7.1 \mathrm{~Hz}, 2 \mathrm{H}), 1.48(\mathrm{t}, J=7.1 \mathrm{~Hz}, 3 \mathrm{H})$; ${ }^{13} \mathrm{C}$ NMR (101 MHz, $\left.\mathrm{CDCl}_{3}\right) \delta 165.5,157.0,154.5,134.9,133.5$, 132.2, 127.9, 124.4, 122.2, 63.8, 14.2; IR (KBr, film, $\left.\mathrm{cm}^{-1}\right)$ 2985, 1748, 1598, 1535, 1441, 1377, 1298, 1253, 1186, 1167, 1100, 1028, 841, 770, 732; HRMS (ESI TOF) calculated for $\mathrm{C}_{11} \mathrm{H}_{9} \mathrm{BrN}_{2} \mathrm{NaO}_{3}(\mathrm{M}$ $+\mathrm{Na})^{+}$318.9689, found $318.9692(1.0 \mathrm{ppm})$.

Ethyl 5-(2-hydroxyphenyl)-1,3,4-oxadiazole-2-carboxylate (4ee V274). This material was obtained from 2-hydroxybenzohydrazide (1e) (152 $\mathrm{mg}, 1.00 \mathrm{mmol}$ ) and ethyl 2-nitroacetate $(6 \mathrm{e})(165 \mu \mathrm{L}, 200 \mathrm{mg}, 1.50 \mathrm{mmol})$. The reaction was carried out at $120-130{ }^{\circ} \mathrm{C}$, and the titled compound was isolated as a colorless oil, $R_{\mathrm{f}} 0.35$ (EtOAc/petroleum ether, $1: 3$ ). Yield $164 \mathrm{mg}(0.70 \mathrm{mmol}, 70 \%) .{ }^{1} \mathrm{H}$ NMR $\left(400 \mathrm{MHz}, \mathrm{CDCl}_{3}\right) \delta 9.92(\mathrm{~s}$, $1 \mathrm{H}), 7.87(\mathrm{dd}, J=7.9,1.6 \mathrm{~Hz}, 1 \mathrm{H}), 7.53-7.46(\mathrm{~m}, 1 \mathrm{H}), 7.14(\mathrm{~d}, J=$ $8.0 \mathrm{~Hz}, 1 \mathrm{H}), 7.06-7.00(\mathrm{~m}, 1 \mathrm{H}), 4.56(\mathrm{q}, J=7.1 \mathrm{~Hz}, 2 \mathrm{H}), 1.48(\mathrm{t}, J$ $=7.2 \mathrm{~Hz}, 3 \mathrm{H}) ;{ }^{13} \mathrm{C} \mathrm{NMR}\left(101 \mathrm{MHz}, \mathrm{CDCl}_{3}\right) \delta 166.2,158.4,155.1$, 154.2, 135.1, 127.4, 120.4, 118.0, 107.2, 63.9, 14.2; IR (KBr, film, $\mathrm{cm}^{-1}$ ) 3245, 2986, 1748, 1625, 1542, 1493, 1377, 1313, 1242, 1186, 1163, 1058, 1021, 852, 751, 710, 676; HRMS (ESI TOF) calculated for $\mathrm{C}_{11} \mathrm{H}_{10} \mathrm{~N}_{2} \mathrm{NaO}_{4}(\mathrm{M}+\mathrm{Na})^{+} 257.0533$, found 257.0532 (0.5 ppm). 


\section{Conflicts of interest}

There are no conflicts to declare.

\section{Acknowledgements}

This work was financed by the Russian Foundation for Basic Research (grant \#18-33-20021 mol_a_ved) grants from the Ministry of Education and Science of the Russian Federation \#4.1196.2017/4.6 and \#4.4589.2017/6.7.

\section{Notes and references}

1 S. Sharma, P. K. Sharma, N. Kumar and R. Dudhe, Pharma. Chemica., 2010, 2, 253-263.

2 H. Khalilullah, M. J. Ahsan, M. Hedaitullah, S. Khan and B. Ahmed, Mini-Rev. Med. Chem., 2012, 12, 789.

3 Y. Zhou, W. H. Wang, W. Dou, X.-L. Tang and W.-S. Liu, Chirality, 2008, 20, 110-114.

4 (a) X. L. Tang, X. H. Peng, W. Dou, J. Mao, J. R. Zheng, W. W. Qin, W.-S. Liu, J. Chang and X.-J. Yao, Org. Lett., 2008, 10, 3653-3656; (b) J. Zhang, L. Zhou, H. A. Al-Attar, K. Shao, L. Wang, D. Zhu, Z. Su, M. R. Bryce and A. P. Monkman, Adv. Funct. Mater., 2013, 23, 4667-4677.

5 See, for example: V. K. Tandon and R. B. Chhor, Synth. Commun., 2001, 31, 1727-1732.

6 See, for example: (a) B. J. Brown, I. R. Clemens and J. K. Neesom, Synlett, 2000, 131-133; (b) P. S. N. Reddy and P. P. Reddy, Indian J. Chem., Sect. B: Org. Chem. Incl. Med. Chem., 1988, 27B, 763-765.

7 (a) K. Du, X. Cao, P. Zhang and H. Zheng, Bioorg. Med. Chem. Lett., 2014, 24, 5318-5320; (b) C. Dobrota, C. C. Paraschivescu, I. Dumitru, M. Matache, I. Baciu and L. L. Ruta, Tetrahedron Lett., 2009, 50, 1886-1888.

8 (a) C. Trecant, A. Dlubala, P. George, P. Pichat, I. Ripoche and Y. Troin, Eur. J. Med. Chem., 2011, 46, 4035-4041; (b) B. Verheyde and W. Dehaen, J. Org. Chem., 2001, 66, 40624064; (c) X. Jiang, R. A. Register, K. A. Killeen, M. E. Thompson, F. Pschenitzka and J. C. Sturm, Chem. Mater., 2000, 12, 2542-2549.

9 (a) N. A. Aksenov, A. V. Aksenov, O. N. Nadein, D. A. Aksenov, A. N. Smirnov and M. Rubin, RSC Adv. , 2015, 5, 71620-71626; (b) A. V. Aksenov, N. A. Aksenov, O. N. Nadein and I. V. Aksenova, Synlett, 2010, 2628-2630.

10 (a) N. A. Aksenov, A. V. Aksenov, O. N. Nadein, D. A. Aksenov, A. N. Smirnov and M. Rubin, $R S C A d v$. , 2015, 5, 71620-71626; (b) A. V. Aksenov, A. N. Smirnov, N. A. Aksenov, A. S. Bijieva, I. V. Aksenova and M. Rubin, Org. Biomol. Chem., 2015, 13, 4289-4295; (c) A. V. Aksenov, N. A. Aksenov, D. S. Ovcharov, D. A. Aksenov, G. Griaznov, L. G. Voskressensky and M. Rubin, RSC Adv., 2016, 6, 82425-82431; (d) A. V. Aksenov, D. S. Ovcharov, N. A. Aksenov, D. A. Aksenov, O. N. Nadein and M. Rubin, RSC Adv., 2017, 7, 29927-29932; (e) A. V. Aksenov, N. A. Aksenov, S. V. Scherbakov, A. N. Smirnov, I. V. Aksenova, V. I. Goncharov and M. A. Rubin, Russ. J. Org. Chem., 2017, 53, 1081-1084; (f) A. I. Konovalov, et al., Russ. J. Org. Chem., 2018, 54, 157-371.

11 For reviews, see: (a) R. Ballini and M. Petrini, Adv. Synth. Catal., 2015, 357, 2371-2402; (b) R. Ballini and M. Petrini, Tetrahedron, 2004, 60, 1017-1047.

12 Formation of species $\mathbf{1 4}$ was unambiguously confirmed by high resolution ESI MS analysis of the reaction mixture. See ESI (page S55†) for details.

13 (a) M.-F. Pouliot, L. Angers, J.-D. Hamel and J.-F. Paquin, Org. Biomol. Chem., 2012, 10, 988-993; (b) Q. Gao, S. Liu, X. Wu, J. Zhang and A. Wu, Org. Lett., 2015, 17, 2960-2963.

14 (a) N. Salvanna, G. C. Reddy, B. R. Rao and B. Das, RSC Adv., 2013， 3， 20538-20544; (b) K. K. Gnanasekaran, B. Nammalwar, M. Murie and R. A. Bunce, Tetrahedron Lett., 2014, 55, 6776-6778; (c) T. Kawano, K. Hirano, T. Satoh and M. Miura, J. Am. Chem. Soc., 2010, 132, 69006901; (d) L.-H. Zou, J. Reball, J. Mottweiler and C. Bolm, Chem. Commun., 2012, 48, 11307-11309.

15 (a) J. Dost, M. Heschel and J. Stein, J. Prakt. Chem., 1985, 327, 109-116; (b) G. I. Elliott, J. R. Fuchs, B. S. J. Blagg, H. Ishikawa, H. Tao, Z.-Q. Yuan and D. L. Boger, J. Am. Chem. Soc., 2006, 128, 10589-10595; (c) J. Garfunkle, C. Ezzili, T. J. Rayl, D. G. Hochstatter, I. Hwang and D. L. Boger, J. Med. Chem., 2008, 51, 4392-4403.

16 (a) F. Uhlig, Angew. Chem., 1954, 66, 435-436; (b) A. L. Huhti and P. A. Gartaganis, Can. J. Chem., 1956, 34, 790-797.

17 Y.-D. Park, J.-J. Kim, H.-A. Chung, D.-H. Kweon, S.-D. Cho, S.-G. Lee and Y.-J. Yoon, Synthesis, 2003, 560-564. 